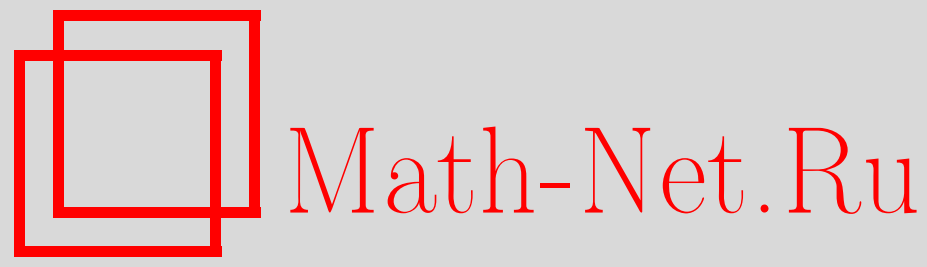

В. С. Буслаев, С. Б. Левин, Система трех трехмерных заряженных квантовых частиц: асимптотическое поведение собственных функций непрерывного спектра на бесконечности, Функи. анализ и его прил., 2012, том 46, выпуск 2, 83-88

DOI: https://doi.org/10.4213/faa3067

Использование Общероссийского математического портала Math$\mathrm{Net.Ru}$ подразумевает, что вы прочитали и согласны с пользовательским соглашением

http://www.mathnet.ru/rus/agreement

Параметры загрузки:

IP : 54.210 .77 .194

26 апреля 2023 г., 15:10:41

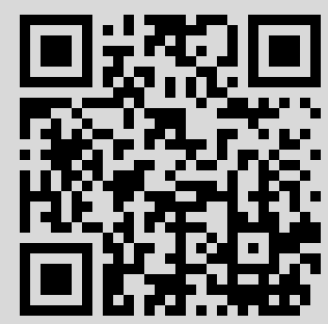


УДК $519.6+517.9$

\title{
Система трех трехмерных заряженных квантовых частиц: асимптотическое поведение собственных функций непрерывного спектра на бесконечности
}

\author{
(C) 2012. В. С. БУСЛАЕВ, С. Б. ЛЕвин
}

Введение. Принципиальные вопросы теории рассеяния в системах двух, трех и большего числа квантовых частиц, рассматриваемые в терминах собственных функций (СФ), исследованы достаточно полно [1], но не исчерпывающе. В этом круге задач имеется набор важных систем, которые до сих пор остаются не исследованными. Мы имеем в виду системы трех и большего числа частиц, взаимодействущих посредством парных кулоновских потенциалов. Имеются два используемых на практике (для компьютерного расчета) подхода к таким задачам. Первый состоит в регуляризации кулоновского потенциала заменой его потенциалом Юкавы. До сих пор, однако, не было возможности оценить эффект такой регуляризации, поскольку никаких достоверных (пусть даже не доказанных строго) результатов для многочастичных систем с кулоновским взаимодействием не было известно.

Второй подход состоит в применении так называемого BBK-приближения к СФ непрерывного спектра, см. [2]. Мы подробнее опишем его позже. Говоря кратко, ВВК-приближение дается явной формулой, которая имеет достаточно быстро убывающую в бесконечности невязку в уравнении. Проблема состоит в том, что на некоторых специальных многомерных направлениях в бесконечности, которые мы назовем экранами, где частицы сближаются попарно, невязка теряет удовлетворительное убывание. Наш результат состоит в модификации BBK-приближения на экранах, после которой модифицированная формула дает удовлетворительное описание асимптотики СФ на всех направлениях в бесконечности. Таким образом, можно сказать, что нам впервые удалось найти асимптотическое поведение СФ. Чтобы оценить смысл этого результата, отметим, что при рассмотрении СФ даже для быстро убывающих потенциалов мы всегда начинаем построения с выписывания асимптотики этих собственных функций (или, что почти эквивалентно) с выписывания соответствующих интегральных уравнений.

Слабые асимптотики. Мы рассматриваем систему трех трехмерных частиц одинаковой массы, взаимодействующих посредством одинаковых парных кулоновских потенциалов. Предположение об одинаковости масс и потенциалов введено лишь для упрощения изложения. Эти ограничения легко снимаются. Вполне очевидно, что система имеет чисто непрерывный спектр, заполняющий полуось $[0, \infty)$, который бесконечнократно вырожден. Традиционный способ идентификации СФ связан с описанием их возможного поведения на бесконечности в конфигурационном пространстве системы. Для быстро убывающих парных потенциалов такая идентификация СФ состоит, например, в выделении 
из их асимптотики в качестве старшего члена плоской волны с заданным волновым вектором q. Для кулоновских потенциалов плоская волна не может быть старшим членом асимптотики СФ в стандартном смысле.

Даже в случае одной частицы, рассеивающейся на кулоновском потенциале $v$, $v(\mathbf{x})=\alpha / x, \mathbf{x} \in \mathbb{R}^{3}, \hat{\mathbf{x}}=\mathbf{x} / x$, такое простое выделение элементарного старшего члена в асимптотическом поведении решения невозможно. Наиболее простое описание может быть дано в терминах так называемой слабой асимптотики [5]. Она представляется следующей формулой [6]:

$$
\psi(\mathbf{x}, \mathbf{k}) \sim \frac{2 \pi i}{k x}\left(\delta(\hat{\mathbf{x}},-\hat{\mathbf{k}}) e^{-i k x+i \eta \ln x}-s_{c}(\hat{\mathbf{x}}, \mathbf{k}) e^{i k x-i \eta \ln x}\right), \quad \eta=\frac{\alpha}{2|\mathbf{k}|}, \quad|\mathbf{x}| \rightarrow \infty
$$

Здесь коэффициент $s_{c}$, фактически матрица рассеяния, в процессе постановки остается не определенным и находится лишь в ходе полного решения задачи. Главной особенностью этого асимптотического описания является то, что мы рассматриваем его в смысле обобщенных функций относительно $\hat{\mathbf{x}}$. Несмотря на ослабленный характер условия, оно благополучно выделяет единственное решение, стандартную собственную функцию.

Чтобы читателю было легче сопоставить эту формулу с классическим асимптотическим описанием собственных функций, отметим, что в слабом смысле $e^{i\langle\mathbf{x}, \mathbf{k}\rangle} \sim \frac{2 \pi i}{k x}\left(\delta(\hat{\mathbf{x}},-\hat{\mathbf{k}}) e^{-i k x}-\delta(\hat{\mathbf{x}}, \mathbf{k}) e^{i k x}\right)$.

Обратимся к системе трех частиц, фиксируем движение центра инерции и будем описывать внутренние движения в терминах переменной $\mathbf{z}$ (подробности ниже). Тогда асимптотика СФ $\Psi(\mathbf{z}, \mathbf{q}), \mathbf{q}$ - волновой вектор, которую можно считать слабым вариантом плоской волны для кулоновских потенциалов, характеризуется формулой

$\Psi(\mathbf{z}, \mathbf{q}) \sim \frac{1}{2}\left(\frac{4 \pi i}{q z}\right)^{5 / 2} \delta(\hat{\mathbf{q}},-\hat{\mathbf{z}}) e^{-i q z+\sum_{j=1}^{3} \eta_{j} \ln z}-\frac{1}{2}\left(\frac{4 \pi i}{q z}\right)^{5 / 2} S_{c}(\mathbf{q}, \hat{\mathbf{z}}) e^{i q z-\sum_{j=1}^{3} \eta_{j} \ln z}$.

Система обозначений для векторов воспроизводит здесь обозначения, использованные в формуле $(1)$, с той разницей, что $\mathbf{z} \in \mathbb{R}^{6}$. Здесь вновь $S_{c}-$ не определенная при постановке задачи функция, которая в конечном счете снова должна оказаться матрицей рассеяния.

Мы определенно верим, что такая характеризация СФ, как и в случае быстро убывающих потенциалов, определяет эти функции единственным образом.

Основной результат работы состоит в том, что, отправляясь от простой асимптотической характеризациии СФ в слабом смысле, мы получаем их асимптотическую характеризачию в традиционном, равномерном, поточечном смысле.

Можно спросить, какую пользу можно извлечь из этого результата. Возможность использовать явную слабую асимптотику для описания решения позволяет, в принципе, формально определить решение. С другой стороны, в работе [3] показано, как можно использовать равномерные асимптотические формулы для численного описания СФ. Это должно быть заведомо более надежно, чем применение юкавовской регуляризации. Кроме того, полученный результат вселяет достаточно убедительную надежду на то, что его можно развить и получить традиционное строгое обоснование того, что существует и единственно решение с полученным здесь асимптотическим поведением. В этой работе мы 
ограничиваемся, однако, эвристическим построением равномерных асимптотических формул для СФ.

Описание модели. Дадим более точное описание модели. Первоначальное конфигурационное пространство системы есть $\mathbb{R}^{9}$. Остановив движение центра инерции, приходим к системе на конфигурационном пространстве $\Gamma=\{\mathbf{z}: \mathbf{z} \in$ $\left.\mathbb{R}^{9}, \mathbf{z}=\left\{\mathbf{z}_{1}, \mathbf{z}_{2}, \mathbf{z}_{3}\right\}, \mathbf{z}_{1}+\mathbf{z}_{2}+\mathbf{z}_{3}=0\right\}$. На $Г$ имеется скалярное произведение $\left\langle\mathbf{z}, \mathbf{z}^{\prime}\right\rangle$, индуцированное скалярным произведением на $\mathbb{R}^{9}$. Система на $Г$ описывается уравнением $H \Psi=\lambda \Psi, \Psi=\Psi(\mathbf{z}) \in \mathbb{C}, \mathbf{z} \in \Gamma, H=-\Delta_{\mathbf{z}}+V(\mathbf{z})$, $V(\mathbf{z})=v\left(\mathbf{x}_{1}\right)+v\left(\mathbf{x}_{2}\right)+v\left(\mathbf{x}_{3}\right), \mathbf{x}_{j} \in \mathbb{R}^{3}$. Здесь $\Delta_{\mathbf{z}}$ - оператор Лапласа на $\Gamma$, $\mathbf{x}_{1}=\frac{1}{\sqrt{2}}\left(\mathbf{z}_{3}-\mathbf{z}_{2}\right), \mathbf{x}_{2}=\frac{1}{\sqrt{2}}\left(\mathbf{z}_{1}-\mathbf{z}_{3}\right), \mathbf{x}_{3}=\frac{1}{\sqrt{2}}\left(\mathbf{z}_{2}-\mathbf{z}_{1}\right)$. Ясно, что $\mathbf{x}_{1}+\mathbf{x}_{2}+\mathbf{x}_{3}=0$. Введем также $\mathbf{y}_{j}=\sqrt{3 / 2} \mathbf{z}_{j}$. Нетрудно убедиться, что на $\Gamma \mathbf{y}_{1}+\mathbf{y}_{2}+\mathbf{y}_{3}=0$, $\mathbf{z}^{2}=\langle\mathbf{z}, \mathbf{z}\rangle=\left\langle\mathbf{x}_{j}, \mathbf{x}_{j}\right\rangle+\left\langle\mathbf{y}_{j}, \mathbf{y}_{j}\right\rangle, j=1,2,3, \Delta_{\mathbf{z}}=\Delta_{\mathbf{x}}+\Delta_{\mathbf{y}}$. Вместе с $\mathbf{z} \in \Gamma$, $\mathbf{x}, \mathbf{y} \in \mathbb{R}^{3}$ будем рассматривать двойственные переменные, импульсы $\mathbf{q} \in \Gamma$, $\mathbf{k}, \mathbf{p} \in \mathbb{R}^{3}$. Мы будем считать, что $v(\mathbf{x})=\alpha / x, \alpha>0$, хотя возможно обобщение на случай $v(\mathbf{x})=\alpha / x+w(\mathbf{x}), x w(\mathbf{x}) \rightarrow 0, x \rightarrow \infty$.

ВВК-приближение. Плоская волна при асимптотическом описании функции $\Psi(\mathbf{z}, \mathbf{q})$ вне окрестностей экранов $\sigma_{j}=\left\{\mathbf{z} \in \Gamma, \mathbf{x}_{j}=0\right\}, j=1,2,3$, должна быть заменена ВBK-приближением $\Psi^{\mathrm{BBK}}(\mathbf{z}, \mathbf{q})$. Это приближение было исследовано в [2], см. также [6], хотя использовалось и ранее в [7], [8]. Оно имеет вид

$$
\Psi^{\mathrm{BBK}}(\mathbf{z}, \mathbf{q}) \sim N_{0} e^{i\langle\mathbf{z}, \mathbf{q}\rangle} D\left(\mathbf{x}_{1}, \mathbf{k}_{1}\right) D\left(\mathbf{x}_{2}, \mathbf{k}_{2}\right) D\left(\mathbf{x}_{3}, \mathbf{k}_{3}\right) .
$$

Здесь $D(\mathbf{x}, \mathbf{k})=\Phi(-i \eta, 1, i x k-i\langle\mathbf{x}, \mathbf{k}\rangle), \mathbf{x}, \mathbf{k} \in \mathbb{R}^{3}, \eta=\alpha /(2 k), \Phi$ - вырожденная гипергеометрическая функция, см. [4]. Постоянная $N_{0}=\prod_{j=1}^{3} N_{c}^{(j)}$ является произведением нормировочных постоянных трех двухчастичных состояний рассеяния $N_{c}^{(j)}=(2 \pi)^{-3 / 2} e^{-\pi \eta_{j} / 2} \Gamma\left(1+i \eta_{j}\right)$.

Стоит отметить, что функция

$$
\psi_{c}(\mathbf{x}, \mathbf{k})=N_{c} e^{i\langle\mathbf{x}, \mathbf{k}\rangle} D(\mathbf{x}, \mathbf{k})
$$

удовлетворяет уравнению $-\Delta_{\mathbf{x}} \psi_{c}+\frac{\alpha}{|\mathbf{x}|} \psi_{c}=k^{2} \psi_{c}$. Решение $\psi_{c}$ является классическим решением задачи рассеяния частицы на кулоновском потенциале.

Невязка ВВК-приближения $Q\left[\Psi^{\mathrm{BBK}}\right]$ вычисляется без труда:

$$
\begin{aligned}
Q\left[\Psi^{\mathrm{BBK}}\right]=- & k_{2} k_{3}\left\langle\hat{\mathbf{k}}_{2}-\hat{\mathbf{x}}_{2}, \hat{\mathbf{k}}_{3}-\hat{\mathbf{x}}_{3}\right\rangle \Phi_{1} \Phi_{2}^{\prime} \Phi_{3}^{\prime} \\
& \quad-k_{3} k_{1}\left\langle\hat{\mathbf{k}}_{1}-\hat{\mathbf{x}}_{1}, \hat{\mathbf{k}}_{3}-\hat{\mathbf{x}}_{3}\right\rangle \Phi_{1}^{\prime} \Phi_{2} \Phi_{3}^{\prime}-k_{1} k_{2}\left\langle\hat{\mathbf{k}}_{2}-\hat{\mathbf{x}}_{2}, \hat{\mathbf{k}}_{1}-\hat{\mathbf{x}}_{1}\right\rangle \Phi_{1}^{\prime} \Phi_{2}^{\prime} \Phi_{3},
\end{aligned}
$$

где ' обозначает производную по последнему аргументу функции Ф. При $z \rightarrow \infty$ вне некоторых окрестностей экранов невязка убывает быстрее кулоновского потенциала. Хотя ВВК-приближение непрерывно вплоть до экранов, его невязка на экранах убывает не быстрее кулоновского потенциала. Поэтому ВВК-приближение не работает в окрестностях экранов.

Разделение переменных. В окрестности каждого экрана уравнение Шрёдингера допускает серьезное упрощение, после которого становится возможным разделение переменных. Например, полный потенциал $V(\mathbf{z})=v\left(\mathbf{x}_{1}\right)+v\left(\mathbf{x}_{2}\right)+$ $v\left(\mathbf{x}_{3}\right)$ в окрестности экрана $\sigma_{1}$ упрощается за счет формул $\mathbf{x}_{2}=-\frac{\sqrt{3}}{2} \mathbf{y}-\frac{1}{2} \mathbf{x}$, $\mathbf{x}_{3}=\frac{\sqrt{3}}{2} \mathbf{y}-\frac{1}{2} \mathbf{x}, \mathbf{x}=\mathbf{x}_{1}, \mathbf{y}=\mathbf{y}_{1}$. В окрестности экрана $\sigma_{1} y \gg 1, y \gg x ;$ 
поэтому формула $V \sim v(x)+v_{m}(y), v_{m}=\frac{4 \alpha / \sqrt{3}}{y}$, дает хорошее приближение к потенциалу. Уравнение с таким потенциалом допускает разделение переменных: $-\Delta_{\mathbf{z}} \chi+\left(v(x)+v_{m}(y)\right) \chi=\lambda \chi$.

Поскольку мы заинтересованы в ограниченных решениях, для $\chi$ естественно возникает следующее представление:

$$
\begin{aligned}
\chi(\mathbf{x}, \mathbf{y}, \mathbf{k}, \mathbf{p})=\int \psi_{c}\left(\mathbf{x}, \mathbf{k}^{\prime}\right) \psi_{m}\left(\mathbf{y}, \mathbf{p}^{\prime}\right) \delta\left(k^{\prime 2}+p^{\prime 2}-E\right) R\left(\mathbf{q}, \mathbf{q}^{\prime}\right) d \mathbf{k}^{\prime} d \mathbf{p}^{\prime} \\
\mathbf{q}=(\mathbf{k}, \mathbf{p}), \mathbf{q}^{\prime}=\left(\mathbf{k}^{\prime}, \mathbf{p}^{\prime}\right) .
\end{aligned}
$$

Здесь $\psi_{c}$ - решение задачи рассеяния для потенциала $v$, а $\psi_{m}$ - решение задачи рассеяния для потенциала $v_{m}$.

Мы можем сформулировать теперь цель настоящей работы более конкретно. Она состоит в том, чтобы дать явное описание старшего порядка асимптотики $\Psi$ в равномерной топологии относительно угловой переменной, $\hat{\mathbf{z}}$. Именно эта задача решена здесь на эвристическом уровне. Заметим, что, имея этот результат, можно изменить постановку задачи (мы предполагаем при этом, что вектор q лежит вне некоторых малых окрестностей трех экранов) и отыскивать решение, характеризуя его равномерной асимптотикой.

ВВК-приближение вблизи экрана. ВВК-приближение вблизи, скажем, экрана $\sigma_{1}$, но, однако, в области, где невязка этого приближения убывает все еще быстрее потенциала, естественно распадается в произведение:

$$
\begin{gathered}
\Psi^{\mathrm{BBK}}(\mathbf{z}, \mathbf{q})=\psi_{c}\left(\mathbf{x}_{1}, \mathbf{k}_{1}\right) \Psi_{1}(\mathbf{z}, \mathbf{q}) \\
\Psi_{1}(\mathbf{z}, \mathbf{q})=N_{0}^{(23)} e^{i\left\langle\mathbf{y}_{1}, \mathbf{p}_{1}\right\rangle} D\left(\mathbf{x}_{2}, \mathbf{k}_{2}\right) D\left(\mathbf{x}_{3}, \mathbf{k}_{3}\right), \quad N_{0}^{(23)}=N_{c}^{(2)} N_{c}^{(3)} .
\end{gathered}
$$

Вблизи экрана переменные $\mathbf{x}_{1}, \mathbf{y}_{1}$ асимптотически имеют разный порядок, $y_{1} \gg x_{1}$. Вычислим в этих предположениях слабую асимптотику $\Psi_{1}(\mathbf{z}, \mathbf{q})$ при $y_{1} \rightarrow \infty$

$$
\begin{aligned}
\Psi_{1}(\mathbf{z}, \mathbf{q})= & \delta\left(\hat{\mathbf{p}}_{1},-\hat{\mathbf{y}}_{1}\right) B_{0}(\mathbf{q}) \frac{2 \pi}{i y_{1} p_{1}} e^{-i y_{1} p_{1}+i \omega \ln y_{1}} e^{i \eta_{2} \ln \left[Z_{2}^{-}+\frac{1}{2} \frac{x_{1}}{y_{1}} V_{2}^{-}\right]} e^{i \eta_{3} \ln \left[Z_{3}^{-}+\frac{1}{2} \frac{x_{1}}{y_{1}} V_{3}^{-}\right]} \\
& -\delta\left(\hat{\mathbf{p}}_{1}, \hat{\mathbf{y}}_{1}\right) B_{0}(\mathbf{q}) \frac{2 \pi}{i y_{1} p_{1}} e^{i y_{1} p_{1}+i \omega \ln y_{1}} e^{i \eta_{2} \ln \left[Z_{2}^{+}+\frac{1}{2} \frac{x_{1}}{y_{1}} V_{2}^{+}\right]} e^{i \eta_{3} \ln \left[Z_{3}^{+}+\frac{1}{2} \frac{x_{1}}{y_{1}} V_{3}^{+}\right]} .
\end{aligned}
$$

Мы использовали здесь следующие обозначения: $Z_{2}^{ \pm}=\frac{\sqrt{3}}{2}\left(1 \pm\left\langle\hat{\mathbf{p}_{1}}, \hat{\mathbf{k}}_{2}\right\rangle\right), Z_{3}^{ \pm}=$ $\frac{\sqrt{3}}{2}\left(1 \mp\left\langle\hat{\mathbf{p}}_{1}, \hat{\mathbf{k}}_{3}\right\rangle\right), V_{2}^{ \pm}=\left\langle\hat{\mathbf{x}}_{1}, \hat{\mathbf{k}}_{2} \pm \hat{\mathbf{p}}_{1}\right\rangle, V_{3}^{ \pm}=\left\langle\hat{\mathbf{x}}_{1}, \hat{\mathbf{k}}_{3} \mp \hat{\mathbf{p}}_{1}\right\rangle, \omega=\frac{\alpha}{2 k_{2}}+\frac{\alpha}{2 k_{3}}$, $B_{0}(\mathbf{q})=B_{0}(\mathbf{q})=-(2 \pi)^{-3} k_{2}^{i \eta_{2}} k_{3}^{i \eta_{3}}$. Коэффициенты $Z_{2(3)}^{+}, V_{2(3)}^{+}$отличаются от коэффициентов $Z_{2(3)}^{-}, V_{2(3)}^{-}$заменой вектора $\hat{\mathbf{p}}_{1}$ на $-\hat{\mathbf{p}}_{1}$.

Вычисление коэффициента $\boldsymbol{R}$. Нам, естественно, понадобится асимптотическое поведение $\chi$ при $y \rightarrow \infty$. Подставим в интеграл $\chi$ слабую асимптотику функции $\psi_{m}$ :

$$
\begin{aligned}
\chi \sim \int \psi_{c}(\mathbf{x}, \mathbf{k}) \frac{2 \pi i}{p y}\left(\delta(\hat{\mathbf{y}},-\hat{\mathbf{p}}) e^{-i p y+i \eta_{m} \ln y}\right. & \left.-S_{m}(\hat{\mathbf{y}}, \mathbf{p}) e^{i p y-i \eta_{m} \ln y}\right) \\
& \times \delta\left(k^{\prime 2}+p^{\prime 2}-E\right) R\left(\mathbf{q}, \mathbf{q}^{\prime}\right) d \mathbf{k}^{\prime} d \mathbf{p}^{\prime} .
\end{aligned}
$$


Здесь $S_{m}$ - кулоновская матрица рассеяния, соответствующая потенциалу $v_{m}$, т. е. параметру $\eta_{m}$.

Дальнейшее асимптотическое упрощение интеграла $\chi$ при $y \rightarrow \infty$ требует информации о структуре коэффициента $R$. Она может быть получена из сопоставления слабых асимптотик по переменной у (непосредственное поточечное сопоставление оказывается значительно более сложной задачей) интеграла (6) и ВBК-приближения (4)-(5) в области, где невязка обоих приближений убывает быстрее потенциала. Это довольно громоздкое вычисление, которое мы не в силах продемонстрировать в настоящей заметке. Но оно является наиболее ответственной частью работы. Основная идея вычисления, однако, довольно естественна. Представление ВВК-приближения на некотором удалении от экрана интегралом $\chi$ есть, фактически, спектральное разложение по собственным функциям $\psi_{m}$, и, таким образом, коэффициент разложения, т. е. коэффициент $R$, находится явно.

Приведем результат:

$$
\begin{aligned}
R\left(\mathbf{q}, \mathbf{q}^{\prime}\right)= & \frac{1}{k p k^{\prime} p^{\prime}} A_{\text {in }}(\mathbf{q}) \frac{\delta\left(\hat{\mathbf{p}}, \hat{\mathbf{p}}^{\prime}\right)}{\left(p^{\prime}-p-i 0\right)^{1+i a}} \delta\left(\hat{\mathbf{k}}, \frac{\hat{\mathbf{k}}+\left(p^{\prime}-p\right) \mathbf{B}_{\text {in }}}{\left|\hat{\mathbf{k}}+\left(p^{\prime}-p\right) \mathbf{B}_{\text {in }}\right|}\right) \\
& +\frac{1}{k p k^{\prime} p^{\prime}} A_{\text {out }}(\mathbf{q}) \frac{G\left(\hat{\mathbf{p}}^{\prime}, \mathbf{p}\right)}{\left(p^{\prime}-p+i 0\right)^{1+i b}} \delta\left(\hat{\mathbf{k}}^{\prime}, \frac{\hat{\mathbf{k}}+\left(p^{\prime}-p\right) \mathbf{B}_{\text {out }}}{\left|\hat{\mathbf{k}}+\left(p^{\prime}-p\right) \mathbf{B}_{\text {out }}\right|}\right) .
\end{aligned}
$$

Здесь ядро $G=S_{m}^{-1}$ удовлетворяет уравнению $\int S_{m}\left(\hat{\mathbf{y}}, p \hat{\mathbf{p}}^{\prime}\right) G\left(\hat{\mathbf{p}}^{\prime}, \mathbf{p}\right) d \hat{\mathbf{p}}^{\prime}=\delta(\hat{\mathbf{y}}, \hat{\mathbf{p}})$,

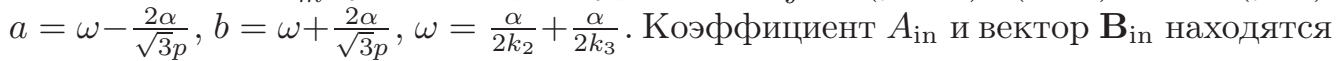
из уравнений $A_{\text {in }}=-\frac{k}{\pi i} \Gamma(1-i a) e^{\frac{\pi a}{2}} B_{0}^{\text {in }}, \mathbf{B}_{\text {in }}=\frac{p}{k^{2}} \hat{\mathbf{k}}-\frac{1}{a k} \Omega_{\text {in }},\left\langle\mathbf{B}_{\text {in }}, \hat{\mathbf{k}}\right\rangle=0$. Со-

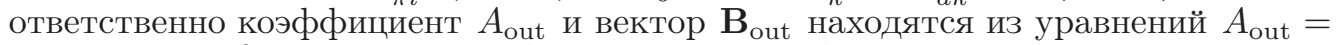
$\frac{k}{\pi i} \Gamma(1-i b) e^{-\frac{\pi b}{2}} B_{0}^{\text {out }}, \mathbf{B}_{\text {out }}=\frac{p}{k^{2}} \hat{\mathbf{k}}-\frac{1}{b k} \Omega_{\text {out }},\left\langle\mathbf{B}_{\text {out }}, \hat{\mathbf{k}}\right\rangle=0$. Мы использовали здесь обозначения

$$
\begin{aligned}
B_{0}^{\text {in }}(\mathbf{q}) & =(2 \pi)^{-3}\left[\frac{\sqrt{3}}{2}\left(1-\left\langle\hat{\mathbf{p}}, \hat{\mathbf{k}}_{2}\right\rangle\right)\right]^{i \eta_{2}}\left[\frac{\sqrt{3}}{2}\left(1+\left\langle\hat{\mathbf{p}}, \hat{\mathbf{k}}_{3}\right\rangle\right)\right]^{i \eta_{3}} k_{2}^{i \eta_{2}} k_{3}^{i \eta_{3}}, \\
B_{0}^{\text {out }}(\mathbf{q}) & =(2 \pi)^{-3}\left[\frac{\sqrt{3}}{2}\left(1+\left\langle\hat{\mathbf{p}}, \hat{\mathbf{k}}_{2}\right\rangle\right)\right]^{i \eta_{2}}\left[\frac{\sqrt{3}}{2}\left(1-\left\langle\hat{\mathbf{p}}, \hat{\mathbf{k}}_{3}\right\rangle\right)\right]^{i \eta_{3}} k_{2}^{i \eta_{2}} k_{3}^{i \eta_{3}}, \\
\Omega_{\text {in }} & =\frac{1}{\sqrt{3}}\left(\eta_{2} \frac{\hat{\mathbf{k}}_{2}-\hat{\mathbf{p}}}{1-\langle\hat{\mathbf{p}}, \hat{\mathbf{k}} 2\rangle}+\eta_{3} \frac{\hat{\mathbf{k}}_{3}+\hat{\mathbf{p}}}{1+\left\langle\hat{\mathbf{p}}, \hat{\mathbf{k}}_{3}\right\rangle}\right), \\
\Omega_{\text {out }} & =\frac{1}{\sqrt{3}}\left(\eta_{2} \frac{\hat{\mathbf{k}}_{2}+\hat{\mathbf{p}}}{1+\left\langle\hat{\mathbf{p}}, \hat{\mathbf{k}}{ }_{2}\right\rangle}+\eta_{3} \frac{\hat{\mathbf{k}}_{3}-\hat{\mathbf{p}}}{1-\left\langle\hat{\mathbf{p}}, \hat{\mathbf{k}}_{3}\right\rangle}\right) .
\end{aligned}
$$

При таком выборе $R$ асимптотическая в слабом смысле формула (6) для $\chi_{j}$ и асимптотическая формула (4)-(5) для $\Psi_{j}^{\mathrm{BBK}}$ при $y_{j} \rightarrow \infty$ совпадают в старшем порядке в пересечении областей

$$
V_{0 j}=\left\{\mathbf{z}: x_{j}<y_{j}^{\nu}, 0<\nu<1\right\}, \quad V_{1 j}=\left\{\mathbf{z}: x_{j}>y_{j}^{\mu}, 1 / 2<\mu<\nu\right\} .
$$

Следующим шагом является получение слабых асимптотик интеграла $\chi$ (см. (6)) при $y \gg 1$ и произвольных $x, x \ll y$, и восстановление по слабым поточечных асимптотик в окрестностях экранов:

$$
\chi(\mathbf{z}, \mathbf{q}) \sim N_{0} e^{i\langle\mathbf{z}, \mathbf{q}\rangle} D\left(\mathbf{x}_{1}, \mathbf{k}_{1}\right) D\left(\tilde{\mathbf{x}}_{2}, \mathbf{k}_{2}\right) D\left(\tilde{\mathbf{x}}_{3}, \mathbf{k}_{3}\right) .
$$


Это выражение совпадает с выражением (4) для $\Psi^{\text {ВВК }}$ с заменой

$$
\begin{gathered}
\mathbf{x}_{2}=-\frac{\sqrt{3}}{2} \mathbf{y}-\frac{1}{2} \mathbf{x} \longrightarrow \tilde{\mathbf{x}}_{2}=-\frac{\sqrt{3}}{2} \mathbf{y}+i \frac{1}{2} \frac{\nabla_{\mathbf{k}} \psi_{c}(\mathbf{x}, \mathbf{k})}{\psi_{c}(\mathbf{x}, \mathbf{k})}, \\
\mathbf{x}_{3}=\frac{\sqrt{3}}{2} \mathbf{y}-\frac{1}{2} \mathbf{x} \longrightarrow \tilde{\mathbf{x}}_{3}=\frac{\sqrt{3}}{2} \mathbf{y}+i \frac{1}{2} \frac{\nabla_{\mathbf{k}} \psi_{c}(\mathbf{x}, \mathbf{k})}{\psi_{c}(\mathbf{x}, \mathbf{k})}
\end{gathered}
$$

где функции $\psi_{c}(\mathbf{x}, \mathbf{k})$ определены в уравнении (3). Этот результат является центральным. Заметим, что при больших $x$ выражения $\tilde{\mathbf{x}}_{2(3)}$ переходят в обыкновенные выражения $\mathbf{x}_{2(3)}$, а интеграл (8) переходит в $\Psi^{\mathrm{BBK}}$ (см. (4)). Заметим также, что вне окрестностей парных направлений рассеяния вперед $\left(\left\langle\hat{\mathbf{x}}_{j}, \hat{\mathbf{k}}_{j}\right\rangle=1\right.$, $j=1,2,3)$ наш результат согласуется с результатами работы [9]. Равномерное по углам описание старшего члена асимптотики решения задачи рассеяния получено впервые.

Формулировка результата. Множества $V_{0}=\Gamma \backslash \bigcup_{j=1}^{3} V_{0 j}$ и $V_{1}=\bigcup_{j=1}^{3} V_{0 j}$ накрывают $\Gamma: \Gamma=V_{0} \cup V_{1}$. Рассмотрим разбиение единицы $1=\zeta_{0}(x, y)+$ $\sum_{j=1}^{3} \zeta_{0 j}(x, y)$, подчиненное этому покрытию. Будем считать, что там, где функции $\zeta_{0 j}, \zeta_{0}$ отличны от постоянных, они зависят от отношения $\rho=\ln x_{j} / \ln y_{j}$.

Составим выражение

Определим на Г функцию

$$
\Psi_{j}^{a s}=\zeta_{0 j} \chi_{j}
$$

$$
\Psi^{a s}=\sum_{j=1}^{3} \Psi_{j}^{a s}+\zeta_{0} \Psi^{\mathrm{BBK}} .
$$

Мы верим в справедливость следующего основного утверждения:

Функция $\Psi^{\text {as }}$ правильно описьвает асимптотическое поведения решения $\Psi$ в старшем порядке в равномерной топологии.

Можно также убедиться, что невязка $Q\left[\Psi^{a s}\right]=-\Delta_{\mathbf{z}} \Psi^{a s}+V \Psi^{a s}-\lambda \Psi^{a s}$ убывает при $z \rightarrow \infty$ быстрее кулоновского потенциала.

\section{ЛитерАтУРА}

[1] Л. Д. Фаддеев, Математические вопросы квантовой теории рассеяния для системы трех частиц, в кн.: Труды МИАН СССР, т. 69, Изд-во АН СССР, М., 1963. [2] M. Brauner, J. S. Briggs, H. Klar, J. Phys. B, 22 (1989), 2265-2287. [3] V. S. Buslaev, S. B. Levin, P. Neittaannmäki, T. Ojala, J. Phys. A: Math. Theor., 43:28 (2010), Article ID 285205. [4] И. С. Градштейн, И. М. Рыжик, Таблищы интегралов, сумм, рядов и произведений, Физматгиз, М., 1963. [5] В. С. Буслаев, в кн.: Проблемы математической физики (Спектральная теория и волновые процессы), т. 1, Изд-во Ленинград. ун-та, 1966, 82-101. [6] С. П. Меркурьев, Л. Д. Фаддеев, Квантовая теория рассеяния для систем нескольких частии, Наука, М., 1985. [7] C. R. Garibotti, J. E. Miraglia, Phys. Rev. A, 21:2 (1980), 572-580. [8] A. L. Godunov, Sh. D. Kunikeev, V. N. Mileev, V. S. Senashenko, in: Proc. 13th Int. Conf. on Physics of Electronic and Atomic Collisions (Berlin), North Holland, Amsterdam, 1983, Abstracts, p. 380. [9] Э. О. Альт, А. М. Мухамеджанов, Письма в ЖЭТФ, 56:9 (1992), 450-454. 